\title{
A FIRST APPROACH TOWARDS BAYESIAN ESTIMATION OF TURBULENT PLASMA PROPERTIES FROM REFLECTOMETRY
}

\author{
G. Hornung ${ }^{1}$, A. Shabbir ${ }^{1}$, G. Verdoolaege ${ }^{1,2}$ \\ ${ }^{1}$ Department of Applied Physics, Ghent University, \\ Sint-Pietersnieuwstraat 41, B-9000 Ghent, Belgium \\ ${ }^{2}$ Laboratory for Plasma Physics - Royal Military Academy \\ (LPP - ERM/KMS), B-1000 Brussels, Belgium
}

September 6, 2016

\section{Corresponding author:}

G. Hornung, Department of Applied Physics, Ghent University

Sint-Pietersnieuwstraat 41, 9000 Ghent, Belgium

E-mail: gregoire.hornung@ugent.be

Tel.: +329264 3587

Fax: +3292644198

Total number of pages: 32

Number of tables: 0

Number of figures: 6

This submission is part of the 1st IAEA Technical Meeting on Fusion Data Processing, Validation and Analysis. The corresponding oral contribution is O-41. 


\title{
A FIRST APPROACH TOWARDS BAYESIAN ESTIMATION OF TURBULENT PLASMA PROPERTIES FROM REFLECTOMETRY
}

\author{
G. Hornung ${ }^{1}$, A. Shabbir ${ }^{1}$, G. Verdoolaege ${ }^{1,2}$ \\ ${ }^{1}$ Department of Applied Physics, Ghent University, \\ Sint-Pietersnieuwstraat 41, B-9000 Ghent, Belgium \\ ${ }^{2}$ Laboratory for Plasma Physics - Royal Military Academy \\ (LPP - ERM/KMS), B-1000 Brussels, Belgium
}

September 6, 2016

\begin{abstract}
The possibility of inferring the properties of electron density fluctuations in tokamak plasmas from a reflectometer signal by means of Bayesian methods is investigated. Within the physical optics approximation the interaction of the probing beam with the plasma is described as reflection from a surface with stochastic properties that is simulated numerically. A Bayesian technique is outlined to solve the inverse problem to determine the surface characteristics from the power spectrum of the reflectometer signal. It is shown that satisfactory estimates of the length and time scales and the amplitude of
\end{abstract}


density fluctuations can be obtained, in conditions relevant to core tokamak plasmas. 


\section{INTRODUCTION}

Anomalous transport of energy and particles in fusion plasmas is related to the complex interaction of fluctuating fields such as temperature, potential and density. Turbulent plasma phenomena are still poorly understood, which is partly due to the difficulty of measuring fluctuating quantities in hot magnetized plasmas. Only a few diagnostics are suitable for turbulence studies and among them is reflectometry, a radar-like technique that is widely used to diagnose electron density fluctuations. Offering good spatial and temporal resolution, reflectometry is an affordable diagnostic requiring limited space without in-vessel components. Nevertheless, the interpretation of the fluctuating reflectometer signal remains a challenging task. Due to the absence of a simple relation between the measured signal and the electron density fluctuations, their properties are often considered the same. In general, this assumption is not justified and might therefore lead to unreliable conclusions.

Several works have addressed estimation of electron density fluctuations from reflectometry measurements. A transfer function that relates the radial wave number spectrum of the density fluctuations to that of the reflectometer phase signal was obtained through numerical means in 1D. ${ }^{1,2}$ In addition, an analytical model was developed within the Born approximation to reconstruct the wave number spectrum related to density fluctuations from the reflectometer cross-correlation function. ${ }^{3,4}$ The possibility of estimating the true turbulence correlation length from the reflected signal has long been 
debated upon within the reflectometry community. Depending on plasma conditions, the estimated correlation length can deviate from the true value. ${ }^{5}$ Recently, a full-wave code was used to determine the optimal probing angle for the measurement of the turbulence correlation length with a Doppler reflectometry system. ${ }^{5,6}$

In this paper, we extend previous studies and follow an alternative approach in an attempt to infer the turbulence properties from a reflectometer signal. Our approach is rooted in inverse problem theory and makes use of Bayesian probability theory. In general, an inverse problem can be solved by assessing to which extent a given state of a physical system is consistent with the measured data. Without suitable restrictions on the possible solutions, it is relatively common that many distinct states are equally likely under the observed data. Therefore, various regularization schemes have been proposed in inverse problem theory. Furthermore, since every experimental signal is subject to uncertainties, probability theory provides a logical approach to tackle inverse problems. In particular, Bayesian probability theory (BPT) has the natural ability to solve inverse problems through Bayes' theorem. In this approach, the number of possible solutions can be restricted as usual through the forward model (e.g. using basis functions for a plasma profile), but also through the so-called prior probability of the parameters of interest. This makes BPT a powerful and flexible framework for solving inverse problems. ${ }^{7,8}$ In addition, a Bayesian approach yields a posterior distribution for the parameters, instead of a single estimate like optimization-based methods. 
As such, the uncertainty of the parameter estimates can be calculated, given the uncertainty on the data. Quantification of these uncertainties is of great importance for the comparison of turbulent simulations with experimental measurements..$^{9,10}$

The main goal of this paper is to investigate numerically the feasibility of the BPT approach to infer turbulent plasma properties from reflectometry signals by considering a simplified plasma model. We first propose a $2 \mathrm{D}$ analytical model for the interaction of the probing reflectometer wave with the electron density fluctuations. Then, a parametrization for the turbulent fluctuations is introduced and Bayes' theorem is used to estimate the poste-

rior probability distribution of a number of parameters that characterize the fluctuations, given the measured reflectometer signal.

\section{PHYSICAL OPTICS MODEL FOR THE PLASMA-WAVE INTERACTION}

In reflectometry, a probing beam is launched and propagates through the plasma until being reflected at the cutoff layer, where the refractive index goes to zero. Reflectometry is a spatially localized diagnostic as most of the plasma-wave interaction takes place close to the cutoff layer. ${ }^{11}$ Indeed, far from the cutoff layer, the probing wave can be considered to propagate in vacuum as the refractive index is close to unity. We take advantage of the separation between the propagation region and the interaction region and 
we assume that the fluctuations of the reflectometer signal originate from electron density fluctuations localized exactly at the cutoff layer. The plasmawave interaction is therefore modeled as the illumination of a corrugated thin conducting surface by a Gaussian probing beam of width $w$ and wave number $k=2 \pi / \lambda$. For the case of conventional reflectometry, i.e. normal incidence and reflection, the physical optics approximation gives a remarkably simple expression for the scattered field ${ }^{12} \rho(t)$ :

$$
\rho(t)=A \int_{-L}^{L} e^{-x^{2} / w^{2}} e^{i 2 k r(x, t)} d x
$$

The scattered field results from the integration along a surface of length $2 L>>w$ of the product of a Gaussian beam function and a term describing the displacement of the cutoff layer $r(x, t)$ due to the presence of density fluctuations. The constant $A$ is used to normalize the scattered field to the field specularly reflected from a flat surface.

As the fluctuations are elongated along the magnetic field lines, the turbulence is almost homogeneous in the toroidal direction over the beam spot size. ${ }^{12}$ Consequently, we have restricted our analysis to the poloidal direction. A schematic representation of the model is shown in Fig. 1, where the $x$-direction approximately corresponds to the poloidal direction in a tokamak.

In this work, the simulations are performed with a time resolution $\Delta t=$ $1.5 \times 10^{5} / f$, in order to mimic a reflectometer operating at $f=50 \mathrm{GHz}$ with a time resolution of $3 \mu \mathrm{s}$. The size of the probing beam is set to $w=5 \lambda \sim 3 \mathrm{~cm}$ 


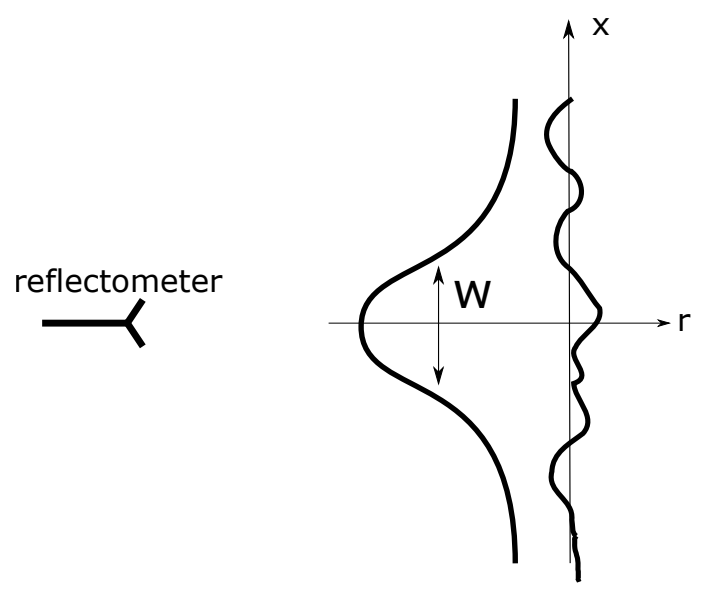

Fig. 1: Schematic representation of the model used to describe the plasmawave interaction through the illumination of a corrugated surface by a Gaussian beam.

which is typical of modern reflectometers. All temporal and spatial quantities are systematically normalized to the time resolution $\Delta t$ and the probing wavelength $\lambda$, respectively.

\section{PARAMETRIZATION OF THE ELEC- TRON DENSITY FLUCTUATIONS}

In our simulation study, the effects of electron density fluctuations on the cutoff layer are represented by a 2D correlated Gaussian random surface defined by three parameters: the amplitude (height) of the fluctuations $h$, together with the correlation length $L_{c}$ and correlation time $t_{c}$ of the fluctuations. $h$ can be understood as the deviation of the cutoff layer with respect to its equi- 
librium position, whereas $L_{c}$ and $t_{c}$ are the characteristic size and time of the fluctuations, respectively. In practice, a surface of length $2 L$ and duration $T$ is obtained in two steps. ${ }^{13}$ First, we generate bivariate random numbers with mean zero and standard deviation $h$. Then, the random numbers are convolved with Gaussian filters in order to obtain the desired correlation properties.

An example of a random surface computed on a grid $N_{x} \times N_{t}=200 \times 2000$ with the parameters $h / \lambda=0.01, t_{c} / \Delta t=5$ and $L_{c} / \lambda=2$ is shown in Fig 2a. The length of the surface was set to $L / \lambda=100$ but we have represented the surface in the interval $x / \lambda=[-15 ; 15]$ in order to reveal the fine scales of the fluctuations.

Figs. 2e and $\mathrm{f}$ show the complex reflectometer signal computed from the random surface and the corresponding power spectrum, respectively. The power spectrum is bell-shaped and has a central peak that corresponds to the specular component of the scattered field. Bell-shaped spectra were observed in several tokamaks, including Tore Supra, Textor and JET, in plasmas dominated by ion temperature gradient turbulence. ${ }^{14}$

The properties of the surface were verified by comparing its statistics against the data model. As expected, Fig. 2c shows that the normalized histogram of the height of the fluctuations is well fitted by a Gaussian distribution with parameters $(\mu, \sigma)=(0, h)$. The averaged autocorrelation functions (ACFs) computed from the data along the $t$ - and $x$-directions are depicted in Figs. $2 \mathrm{~b}$ and $\mathrm{d}$, respectively. To ensure that the ACFs follow the underlying 

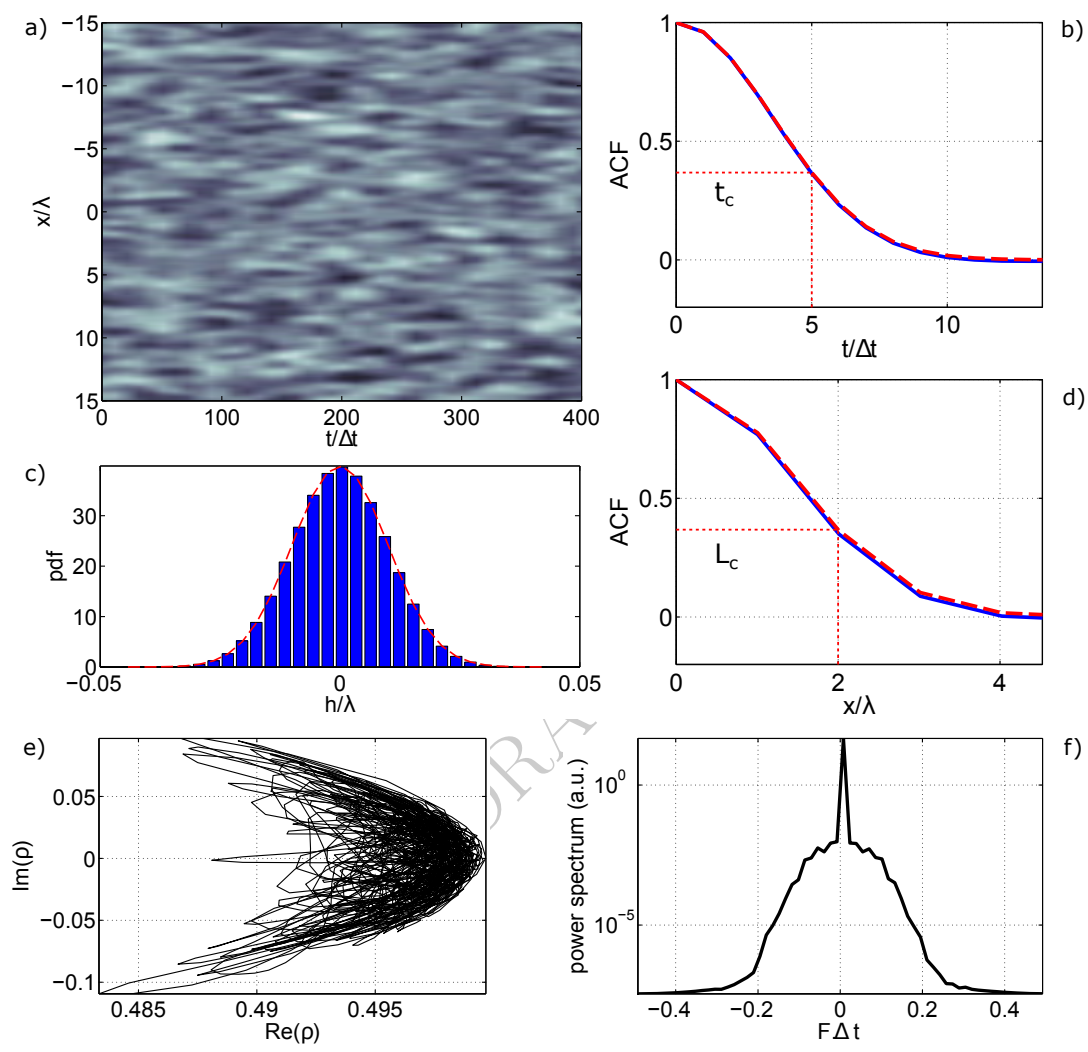

Fig. 2: a) Example of a simulated Gaussian correlated random surface. b) and d) are the autocorrelation function $(\mathrm{ACF})$ of the random surface computed along the $t$ - and $x$-directions, respectively. The corresponding correlation length and time are indicated. c) shows the distribution of the fluctuations. For b) to d), the solid curves represent the data whereas the dashed curves represent the model. e) Complex reflectometer signal associated to the random surface and f) corresponding power spectrum. 
Gaussian model, the proportions of the surface have to be much larger than the scales of the fluctuations, namely $t_{c} / T<<1$ and $L_{c} / 2 L<<1$.

\section{BAYES' THEOREM AND THE SOLU- TION TO THE INVERSE PROBLEM}

Bayes' theorem is a straightforward consequence of the rule of conditional probability. Given two events $A$ and $B$, the probability of $A$ given $B$ is related to the probability of $B$ given $A$ such that $p(A \mid B) p(B)=p(B \mid A) p(A)$. Replacing $A$ by parameters and $B$ by data, Bayes' theorem naturally provides a solution to the inverse problem as it allows us to estimate the probability of a specific set of parameters given the outcome of an experiment. Applied to our problem, Bayes' theorem reads:

$$
\underbrace{p\left(h, t_{c}, L_{c} \mid\left\{d_{i}\right\}, I\right)}_{\text {posterior }}=\underbrace{p\left(\left\{d_{i}\right\} \mid h, t_{c}, L_{c}, I\right)}_{\text {likelihood }} \underbrace{p\left(h, t_{c}, L_{c} \mid I\right)}_{\text {prior }} / \underbrace{p\left(\left\{d_{i}\right\} \mid I\right)}_{\text {evidence }},
$$

where $h, t_{c}$ and $L_{c}$ are the turbulent parameters and $d_{i}$ the measured signal. Any further background information is represented by the symbol $I$, which is sometimes assumed implicitly.

The great benefit of Bayes' theorem lies in the fact that it relates the posterior distribution, which is the solution of the inverse problem, to other terms which are much easier to evaluate. Each term in Eq. 1 bears a spe- 
cific name. In parameter inference problems, the evidence simply acts as a normalizing constant and will therefore be disregarded here. The prior probability is used to encode the knowledge about the physical system that is available before analyzing the data. In this work, we assume that only lower and upper bounds on the parameters are known and, accordingly, we choose a uniform prior in order to give an equal weight to any possible solution $\left\{h, t_{c}, L_{c}\right\}$ within the bounds. In tokamaks, the turbulent time scale is bounded by the inverse of the ion gyrofrequency $\omega_{c i}$ and the confinement time $\tau_{e}$. Similarly, the characteristic size of the density fluctuations should be larger than the ion gyroradius $\rho_{i}$, but smaller than the typical size of the device $a$. The fluctuation height $h$ is obviously positive and is also bounded by the device length scale $a$. The prior distribution is therefore defined as follows:

$p\left(h, t_{c}, L_{c} \mid I\right)=\left\{\begin{array}{cc}\text { cst. } & \text { if } \quad 0 \leq h \leq a, \omega_{c i} \leq t_{c} \leq \tau_{e}, \rho_{i} \leq L_{c} \leq a \\ 0 & \text { otherwise. }\end{array}\right.$

The likelihood, noted $\mathcal{L}(\boldsymbol{\theta} \mid \boldsymbol{d})$ to emphasize the dependence on the system parameters, gives the probability density to observe the measured signal $\boldsymbol{d}$ given the parameters $\boldsymbol{\theta}=\left\{h, t_{c}, L_{c}\right\}$. The likelihood evaluates the difference between the measured signal $\boldsymbol{d}$ and the data simulated with the forward model $F(\boldsymbol{\theta})$. Under the hypothesis that the experimental signal is correctly 
described by the forward model, the likelihood should be large if the measured signal is consistent with the considered set of turbulent parameters, within the measurement error.

Strictly speaking, in this work no signals are measured experimentally since all our experiments are numerical. Instead, we simulate experimental data $\boldsymbol{d}$ by calculating the reflectometer signal associated to a known set of original parameters $\boldsymbol{\theta}^{\mathbf{0}}=\left\{h^{0}, t_{c}^{0}, L_{c}^{0}\right\}$. Our main objective is thus to investigate for which conditions it is possible to correctly infer the original parameters $\boldsymbol{\theta}^{\mathbf{0}}$.

The problem treated here includes an extra complication, compared to most studies of inverse problems, as it contains an inherent stochastic component. Indeed, the turbulent surface is defined as a random process, meaning that two realizations generated with exactly the same original parameters will lead to two different surfaces. As a consequence, the raw reflectometer signal scattered by such surfaces will also show different waveforms. This is illustrated in Fig. 3, where the model signal (solid line) has been simulated with the same parameters as the 'measured' signal (dashed line). Thus, the raw reflectometer signal is not a convenient choice for computation of the likelihood.

To resolve this situation, we consider in the likelihood the power spectrum of the measured reflectometer signal. The power spectrum can be expected to be related in a more-or-less deterministic way to the true parameters of the surface, in contrast to the reflectometer signal itself. As an illustration, 

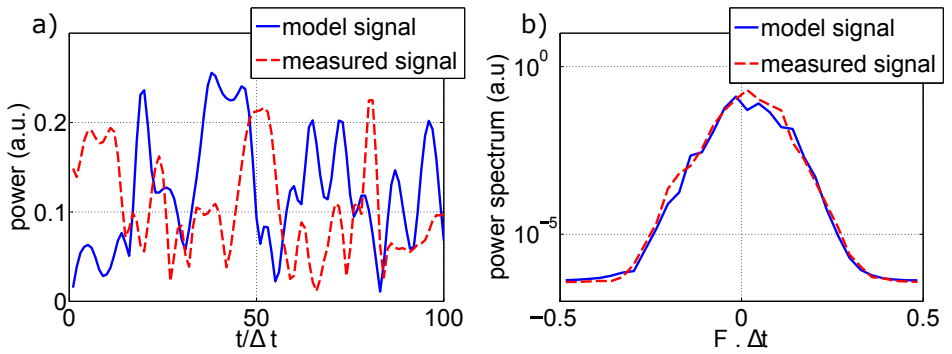

Fig. 3: a) Power signals computed for two realizations of the turbulent surfaces generated with the parameters $h / \lambda=0.2, L_{c} / \lambda=1$ and $t_{c} / \Delta t=10$ and computed on a grid $N_{x} \times N_{t}=200 \times 2000$. The beam width is set to $w / \lambda=5$. The associated power spectra computed from the complex reflectometer signals are shown in b).

Fig. 3b shows the power spectrum computed from the scattered field for the measured and model signals. Contrary to the reflected power represented in Fig. 3 a, the power spectrum is approximately constant if the turbulent parameters are fixed.

\section{IV.A Likelihood of the Power Spectrum}

The choice of the likelihood should be consistent with the random uncertainties affecting the 'measured' and modeled signals. The uncertainties on the power spectrum, that are responsible for the slight differences between the curves depicted in Fig. 3b, have a statistical nature. These uncertainties appear because the time series used for the computation of the spectrum has a finite length. The distribution of the power spectrum $\hat{P}\left(f_{i}\right)^{m}$ at frequency $f_{i}$, computed from the modeled signal and averaged over $N_{\text {win }}$ independent 
windows, sliding over the signal, is given by ${ }^{15}$

$$
2 N_{w i n} \frac{\hat{P}\left(f_{i}\right)^{m}}{P\left(f_{i}\right)^{\text {true }}} \sim \chi^{2}\left(2 N_{w i n}\right) .
$$

Here, $f_{i}$ is any frequency obeying $0<\left|f_{i} \Delta t\right|<1 / 2$ and $P\left(f_{i}\right)^{\text {true }}$ stands for the true power spectrum computed in the limit of an infinitely long time series. Hence, the ratio between the estimated and the true power spectrum follows a $\chi^{2}$ distribution with $2 N_{\text {win }}$ degrees of freedom. The mean and standard deviation of this distribution are given by $\mu=2 N_{w i n}$ and $\sigma=$ $\sqrt{2 N_{\text {win }}}$, respectively. We therefore deduce that the relative uncertainty on the average power spectrum, quantified by $\epsilon^{m}=\sigma / \mu=1 / \sqrt{2 N_{\text {win }}}$, decreases with the square root of the number of windows. We will use a sampling method to estimate the posterior distribution, therefore a large number of model signals will need to be computed. As a result, increasing $N_{\text {win }}$ to reduce $\epsilon^{m}$ quickly becomes computationally prohibitive. In practice, the model power spectra were computed from $N_{t}^{m}=2000$-point time series using the Welch algorithm with a 128-point sliding Hamming window, which leads to a random uncertainty $\epsilon^{m} \approx 18 \%$.

The distribution defined in Eq. 4 measures the difference between the modeled and the true power spectrum, but what is actually required is a measure of the difference between the modeled and the 'measured' power spectrum. Contrary to the modeled power spectrum, in our study the measured power spectrum is computed only once. It is therefore possible to 
estimate the measured power spectrum from a time series of duration $N_{t}^{d}$ that lasts much longer than the model time series. By setting $N_{t}^{d}=100 N_{t}^{m}$, the uncertainty on the average measured spectrum $\epsilon^{d} \approx 1.8 \%$ becomes negligible with respect to $\epsilon^{m}$, allowing us to consider that the measured and the true power spectrum are equal. We will therefore use the likelihood $\mathcal{L}\left(\boldsymbol{\theta} \mid d_{i}\right) \sim \chi^{2}\left(2 N_{\text {win }}\right)$ for the quantity $\hat{P}\left(f_{i}, \boldsymbol{\theta}\right)^{m} / \hat{P}\left(f_{i}\right)^{d}$ which evaluates the ratio of the modeled power spectrum $F_{i}(\theta)=\hat{P}\left(f_{i}, \boldsymbol{\theta}\right)^{m}$ to the measured power spectrum $d_{i}=\hat{P}\left(f_{i}\right)^{d}$ at the frequency $f_{i}$. The full likelihood is given by the product of the individual likelihoods $\mathcal{L}(\boldsymbol{\theta} \mid \boldsymbol{d})=\prod_{i=1}^{N} \mathcal{L}\left(\boldsymbol{\theta} \mid d_{i}\right)$, where $N=16$ frequencies are used to discretize the power spectrum.

The validity of the likelihood was checked by computing 1000 realizations of $\hat{P}\left(f_{i}, \boldsymbol{\theta}\right)^{m}$ for the same parameters $\boldsymbol{\theta}$. Fig. 4 shows that the normalized histogram of $2 N_{w i n} \hat{P}\left(f_{i}, \boldsymbol{\theta}\right)^{m} / \hat{P}\left(f_{i}\right)^{d}$ is indeed correctly represented by the $\chi^{2}$ distribution.

\section{IV.B Sampling the Posterior Distribution}

The posterior distribution for our problem cannot be computed analytically but has to be estimated using numerical methods. Markov Chain Monte Carlo (MCMC) is widely used in Bayesian inference to draw samples from the posterior distribution. ${ }^{16}$ We used a random-walk MCMC algorithm, creating a Markov chain of samples drawn from a so-called proposal distribution. If $\boldsymbol{\theta}^{(t)}$ denotes the current state of the chain at 'time' $t$, then a new sample $\boldsymbol{\theta}^{(t+1)}$ is accepted with probability determined by the ratio of the posterior 


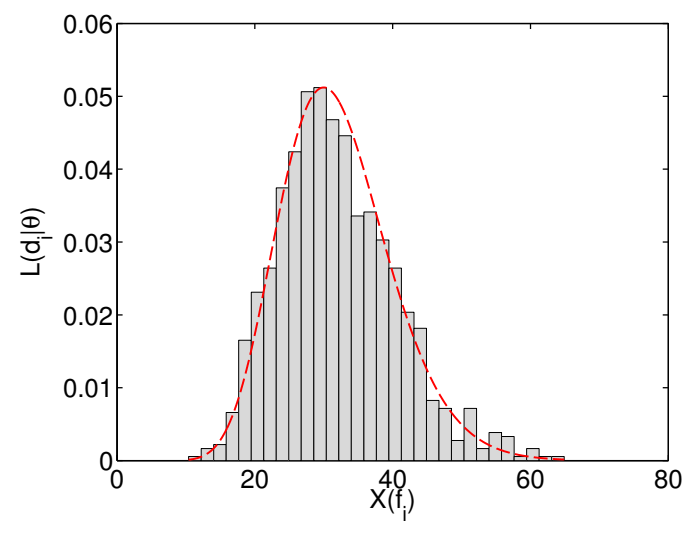

Fig. 4: Fig. 4. Normalized histogram computed for 1000 realizations of the quantity $X\left(f_{i}\right)=2 N_{\text {win }} \hat{P}\left(f_{i}, \boldsymbol{\theta}\right)^{m} / \hat{P}\left(f_{i}\right)^{d}$ for frequency $f_{i} \Delta t=0.156$. The parameters have been set to $\left\{h / \lambda, L_{c} / \lambda, t_{c} / \Delta t\right\}=\{0.01,2,2\}$. The dashed curve represents the theoretical $\chi^{2}$ distribution with $2 N_{\text {win }}=32$ degrees of freedom.

densities $p\left(\boldsymbol{\theta}^{(t+1)} \mid \boldsymbol{d}, I\right) / p\left(\boldsymbol{\theta}^{(t)} \mid \boldsymbol{d}, I\right)$. The sample is always accepted if it increases the posterior density and eventually the chain converges towards the true posterior distribution.

Results obtained in MCMC simulations performed with the original parameters set to $\left\{h^{0} / \lambda, L_{c}^{0} / \lambda, t_{c}^{0} / \Delta t\right\}=\{0.1,4,4\}$ are shown in Fig. 5. The values of the original parameters were chosen to be characteristic of plasma turbulence under tokamak core conditions. Given that the simulations were performed for $\lambda=5.9 \mathrm{~mm}$ and $\Delta t=3 \mu$ s (see Section II), the plasma parameters $L_{c}^{0} / \lambda=4$ and $t_{c}^{0} / \Delta t=4$ translate into a normalized poloidal wave number $k_{\theta} \rho_{i}=0.4$ and an eddy turn-over time $\tau=12 \mu$ s if we consider a deuterium plasma with a magnetic field $B=4 \mathrm{~T}$ and an ion temperature $T_{i}=1 \mathrm{keV}$. The above values are typical for ion temperature gradient or 
trapped electron mode turbulence. ${ }^{17,18}$ The mean cutoff layer displacement $h$ can be translated into the more conventional fluctuation level using a mixing length estimate ${ }^{19} \delta n / n \sim h / L_{n}$. Taking a density gradient scale $L_{n}=30 \mathrm{~cm}$, $h^{0} / \lambda=0.1$ corresponds to a fluctuation level of about $\delta n / n \approx 2 \%$.

In panels $\mathrm{a}-\mathrm{c}$ of Fig. 5, part of the chains obtained with the MCMC method are shown. The so-called burn-in period can be noticed that is needed before the chain starts to converge. A Laplacian proposal distribution was employed, which, thanks to its heavy tails, allows a good chance of large jumps in order to efficiently explore the parameter space. In total, $5 \times 10^{4}$ samples were generated. The first 500 samples were disregarded as they correspond to the burn-in period. About 900 samples were accepted after the burn-in period. These samples were used to estimate the posterior.

The convergence of the chain was investigated using several indicators. The time trace of the posterior, shown in fig. 5g, quickly reaches a steady state, we therefore consider that the chain converges after about 500 samples. Figs. 5i and $\mathrm{h}$ show the cross- $(\mathrm{CCF})$ and the auto-correlation functions (ACF) for the different parameters, respectively. The fluctuations amplitude and time scale are strongly correlated whereas the correlation between the amplitude and length scale as well as the correlation between the length and time scale is rather modest.

For each parameter, the ACF goes rapidly to zero indicating a satisfactory mixing of the chain. The number of independent samples $N_{\text {ind }}$ can be estimated from the ACF properties such that $N_{\text {ind }} \sim N_{a c c} / l_{A C F}$, where $N_{\text {acc }}$ 

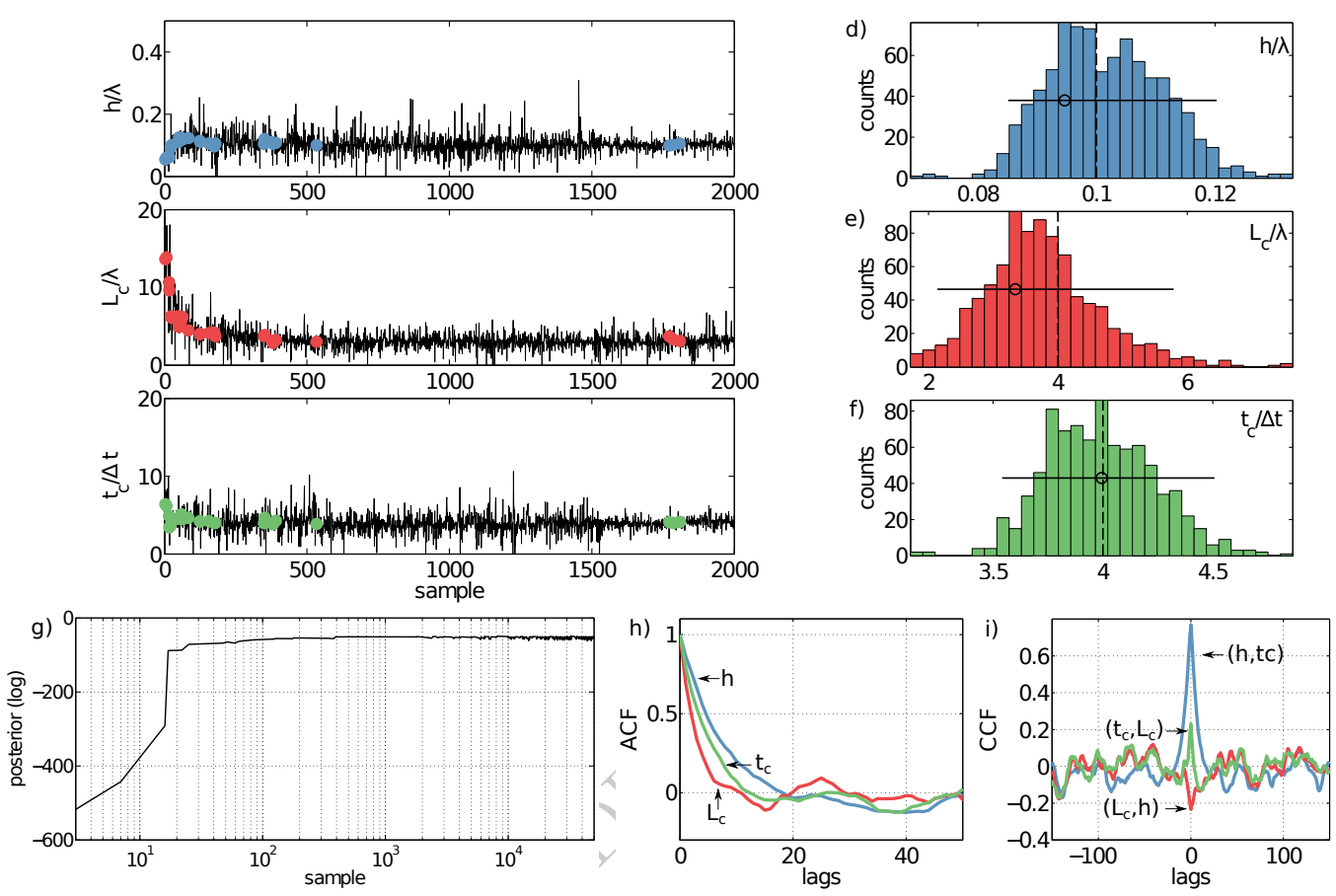

Fig. 5: Fig. 5. Top left panels: parameter samples generated during an MCMC run. The dots in panels (a), (b) and (c) represent the accepted samples for the fluctuation amplitude $h$, characteristic length $L_{c}$ and time $t_{c}$, respectively. The corresponding estimated posterior distributions are represented in the panels (e-f) on the top right. The vertical dashed lines indicate the values of the original parameters. The open circles correspond to the peak values of the posterior distributions whereas the horizontal lines denote the $95 \%$ credible intervals. Bottom panels: diagnostics used for assessing the convergence of the chain. (g) Evolution of the posterior probability. (h) Auto- and (i) cross-correlation functions, each curve is labeled with the corresponding parameters. 
and $l_{A C F}$ are the number of accepted samples and the auto-correlation lag, respectively. The auto-correlation lags were obtained by fitting the ACF with an exponential function. The number of independent samples for the amplitude, length and time scale is about $N_{\text {ind }} \sim 160, N_{\text {ind }} \sim 366$ and $N_{\text {ind }} \sim 226$, respectively.

Histograms obtained from the MCMC run are displayed in panels $\mathrm{d}-\mathrm{f}$ of Fig. 5, for the parameters $h, L_{c}$ and $t_{c}$, respectively. The best estimates for each of the parameters are taken as the peak values (mode) of the posterior distributions, while the error bars are derived from the bounds of the $95 \%$ credible intervals. For instance, the best estimate for the fluctuation amplitude is $h / \lambda=0.946$ and the true value $h^{0} / \lambda$ (in this case $h^{0} / \lambda=0.1$ ) lies with probability 0.95 in the interval $[0.085,0.120]$.

Fig. 5 also reveals that the estimates of the fluctuation amplitude $h$ and time scale $t_{c}$ are more accurate than the estimate of the length scale $L_{c}$. The reason is that the power spectrum depends mostly on the amplitude and time scale of the turbulence. Accordingly, the posterior distribution for $L_{c}$ is, from the relative point of view, wider than the posteriors for $h$ and $t_{c}$. Still, it is possible to fairly well infer the characteristic length of the turbulence. This is remarkable in view of the spatial averaging process related to the finite width of the illuminating beam.

Next, we show the performance of the method for various choices of the original parameter values describing the random surface, covering a range of amplitudes and spatial and temporal scales. The results are presented in 

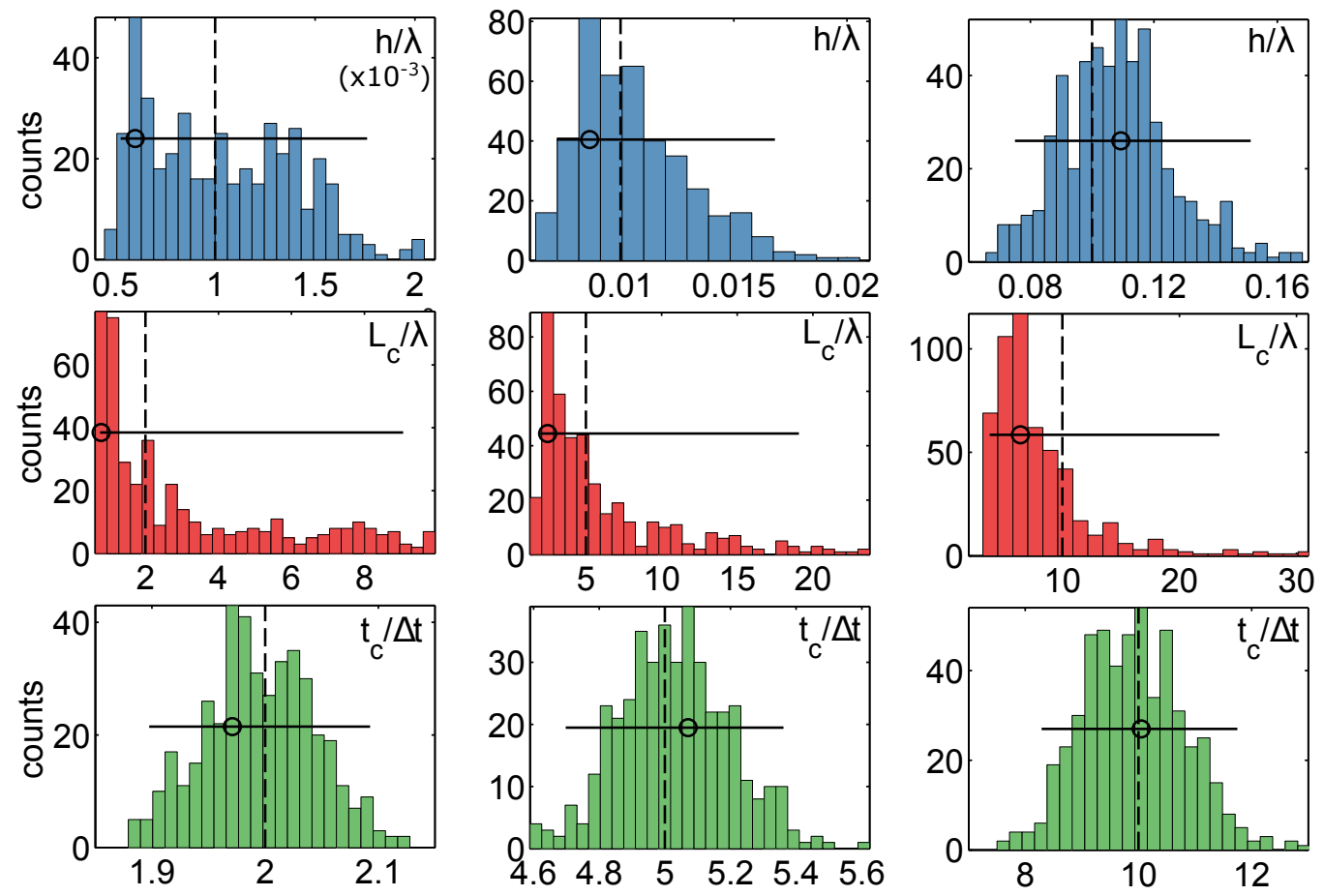

Fig. 6: Fig. 6. Posterior distributions obtained while scanning the original parameters $\left\{h^{0}, L_{c}^{0}, t_{c}^{0}\right\}$. The left, center and right panels were obtained with parameters $\left\{h^{0} / \lambda, L_{c}^{0} / \lambda, t_{c}^{0} / \Delta t\right\}=\left\{10^{-3}, 2,2\right\},\left\{h^{0} / \lambda, L_{c}^{0} / \lambda, t_{c}^{0} / \Delta t\right\}=$ $\left\{10^{-2}, 5,5\right\}$ and $\left\{h^{0} / \lambda, L_{c}^{0} / \lambda, t_{c}^{0} / \Delta t\right\}=\left\{10^{-1}, 10,10\right\}$, respectively. For each posterior, the dashed vertical line shows the value of the original parameter. The best estimates (open circles) and the 95\% credible intervals (horizontal lines) are also shown. 
Fig. 6 where each column corresponds to a different parameter set. The left column of Fig. 6 shows that for very low amplitude fluctuations, it becomes more difficult to infer $h$ and $L_{c}$. The posterior distributions are significantly skewed and the relative error on the best estimate is about $50 \%$. The method works well for a moderate fluctuation amplitude as shown in the center and right columns of Fig. 6. As before, the inferred values of $L_{c}$ are less accurate compared to those of $h$ and $t_{c}$. The method starts to fail if we keep increasing the fluctuation amplitude. From $h^{0} / \lambda \gtrsim 0.5$, the reflectometer signal saturates and as a consequence the power spectrum is no longer dependent on the values of the turbulent parameters.

\section{CONCLUSION AND PERSPECTIVES}

We have proposed a new and innovative approach for the interpretation of a fluctuating reflectometer signal. The approach makes use of Bayesian probability theory to infer the properties of turbulent fluctuations on the basis of the measured reflectometer signal and a priori information. This paper primarily aims at numerically investigating the feasibility of such an approach. We have therefore considered a simplified model where the interaction of the probing beam with correlated Gaussian fluctuations was described in the framework of physical optics. The difficulties posed by the intrinsic stochastic properties of the turbulent fluctuations have been overcome by evaluating the likelihood of the power spectrum instead of the raw reflectometer signal. 
We have been able to infer with a satisfactory accuracy the properties of a simulated fluctuating surface with parameters relevant to tokamak core plasma conditions. The main limiting factor of the method turns out to be the fluctuation amplitude. Indeed, if the turbulent activity is too strong, $h / \lambda \gtrsim 0.5$, the reflectometer signal saturates and our method breaks down. In practice, this might only happen in the vicinity of the boundary between closed and open magnetic field line regions where the fluctuation level is usually very large.

The method is clearly only in its infancy and much work remains to be done before the Bayesian approach can be routinely used to process real fluctuating reflectometer signals. While the assumption of Gaussian fluctuations seems reasonable in core plasmas, ${ }^{20-22}$ the physical optics model used to describe the plasma-wave interaction has a limited domain of application. The simplicity of this model relies on neglecting the presence of the plasma on the wave path. A comparison with more realistic models has suggested that the physical optics model might be a good approximation for O-mode reflectometry in regions where the refractive index gradient is steep. ${ }^{23}$ Nevertheless, the domain of application of the simple model might be extended for X-mode reflectometry, given that the refractive index is generally steeper for X-mode waves than for O-mode waves due to the dependence on the magnetic field.

In the near future, we plan to consider also the effect of instrumental noise on the reflectometer signal. Contrary to the finite length of the signal which leads to stochastic uncertainties, instrumental noise will produce a system- 
atic modification of the statistical quantities derived from the reflectometer signal. As a consequence, we envision to treat instrumental noise as an extra parameter which can also be inferred with the Bayesian approach. The first attempts of including instrumental noise have revealed that it is probably not sufficient to consider only the power spectrum in order to correctly infer the parameters with noisy signals. Additional statistical quantities, such as histograms computed from the complex reflectometer signal, might also bring new and relevant information.

\section{Acknowledgement}

Fruitful discussions with dr. Rainer Fischer held during the 1st IAEA Technical Meeting on Fusion Data Processing, Validation and Analysis are greatly acknowledged. 


\section{References}

[1] L. Vermare et al. "Density Fluctuation Measurements using X-mode Fast Sweep Reflectometry on Tore Supra." Nucl. Fusion, 46, S743 (2006).

[2] T. Gerbaud et al. "Comparison of Density Fluctuation Measurements Between O-mode and X-mode Reflectometry on Tore Supra." Rev. Sci. Instrum., 77, 10E928 (2006).

[3] E. Z. Gusakov and N. V. Kosolapova. "Fluctuation Reflectometry Theory and the Possibility of Turbulence Wave Number Spectrum Reconstruction Using the Radial Correlation Reflectometry Data." Plasma Phys. Control. Fusion, 53, 045012 (2011).

[4] E. Z. Gusakov et al. "2D Modeling of Turbulence Wave Number Spectra Reconstruction from Radial Correlation Reflectometry Data." Plasma Phys. Control. Fusion, 57, 075009 (2015).

[5] E. Blanco and T. Estrada. "Two-dimensional Full-wave Simulations of Radial Correlation Doppler Reflectometry in Linear and Non-linear Regimes." Plasma Phys. Control. Fusion, 55, 125006 (2013).

[6] F. Fernández-Marina et al. "Turbulence Radial Correlation Length Measurements Using Doppler Reflectometry in TJ-II." Nucl. Fusion, 54, 072001 (2014). 
[7] A. Tarantola. Inverse Problem Theory and Methods for Model Parameter Estimation. siam (2005).

[8] U. von Toussaint. "Bayesian Inference in Physics." Rev. Mod. Phys., 83, 943 (2011).

[9] A. CAsati et al. "Turbulence in the TORE SUPRA Tokamak: Measurements and Validation of Nonlinear Simulations." Phys. Rev. Lett., 102, 165005 (2009).

[10] C. Holland et al. "Implementation and Application of Two Synthetic Diagnostics for Validating Simulations of Core Tokamak Turbulence." Phys. Plasmas, 16, 052301 (2009).

[11] E. Mazzucato. "Microwave Reflectometry for Magnetically confined plasmas." Rev. Sci. Instrum., 69, 2201 (1998).

[12] G. Conway. "2D Modelling of Radial Correlation Reflectometry." Plasma Phys. Control. Fusion, 39, 407 (1997).

[13] N. Garcia and E. Stoll. "Monte Carlo Calculation for Electromagnetic-Wave Scattering from Random Rough Surfaces." Phys. Rev. Lett., 52, 1798 (1984).

[14] H. Arnichand et al. "Discriminating the Trapped Electron Modes Contribution in Density Fluctuation Spectra." Nucl. Fusion, 55, 093021 (2015). 
[15] H. von Storch and F. ZwiERs. Statistical Analysis in Climate Research. Chapter 12, p. 271, Cambridge University Press (1999).

[16] K. Mosegand and A. Tarantola. "Monte Carlo Sampling of Solutions to Inverse Problems." Journal of Geophysical Research: Solid Earth, 100, 12431 (1995).

[17] W. Horton. "Drift Waves and Transport." Rev. Mod. Phys., 71, 735 (1999).

[18] X. Garbet et al. "Physics of Transport in Tokamaks." Plasma Phys. Control. Fusion, 46, B557 (2004).

[19] X. Garbet. "Introduction to Turbulent Transport in Fusion Plasmas." C.R. Physique, 7, 573 (2006).

[20] G. Xu et al. "Blob/hole Formation and Zonal-flow Generation in the Edge Plasma of the JET Tokamak." Nucl. Fusion, 49, 092002 (2009).

[21] B. NolD et al. "Generation of Blobs and Holes in the Edge of the ASDEX Upgrade Tokamak." Plasma Phys. Control. Fusion, 52, 065005 (2010).

[22] P. Simon et al. "Scaling and Transport Analyses Based on an International Edge Turbulence Database." Plasma Phys. Control. Fusion, 56, 095015 (2014). 
[23] G. D. Conway et al. "Coupling of Turbulence and Reflectometer Simulation Codes and Comparison with Experiment." Plasma Phys. Control. Fusion, 44, 451 (2002). 